\title{
Angelica Sinensis Polysaccharide Induces Erythroid Differentiation of Human Chronic Myelogenous Leukemia K562 Cells
}

\author{
Lu Wang ${ }^{1}$, Rong Jiang ${ }^{1}$, Shu-Dan Song ${ }^{2}$, Zi-Sen Hua ${ }^{1}$, Jian-Wei Wang ${ }^{1}$, Ya-Ping \\ Wang ${ }^{1 *}$
}

\begin{abstract}
Leukemia is a clonal disorder with blocked normal differentiation and cell death of hematopoietic progenitor cells. Traditional modalities with most used radiation and chemotherapy are nonspecific and toxic which cause adverse effects on normal cells. Differentiation inducing therapy forcing malignant cells to undergo terminal differentiation has been proven to be a promising strategy. However, there is still scarce of potent differentiation inducing agents. We show here that Angelica sinensis polysaccharide (ASP), a major active component in Dong quai (Chinese Angelica sinensis), has potential differentiation inducing activity in human chronic erythromegakaryoblastic leukemia K562 cells. MTT assays and flow cytometric analysis demonstrated that ASP inhibited $\mathrm{K562}$ cell proliferation and arrested the cell cycle at the G0/G1 phase. ASP also triggered K562 cells to undergo erythroid differentiaton as revealed by morphological changes, intensive benzidine staining and hemoglobin colorimetric reaction, as well as increased expression of glycophorin A (GPA) protein. ASP induced redistribution of STAT5 protein from the cytoplasm to the nucleus. Western blotting analysis further identified that ASP markedly sensitized K562 cells to exogenous erythropoietin (EPO) by activating EPO-induced JAK2/ STAT5 tyrosine phosphorylation, thus augmenting the EPO-mediated JAK2/STAT5 signaling pathway. On the basis of these findings, we propose that ASP might be developed as a potential candidate for chronic myelogenous leukemia inducing differentiation treatment.
\end{abstract}

Keywords: Angelica sinensis polysaccharides - K562 - inducing differentiation - JAK2 - STAT5

Asian Pac J Cancer Prev, 16 (9), 3715-3721

\section{Introduction}

Leukemia is a clonal disorder that results as a consequence of an acquired somatic mutation in hematopoietic progenitor cells that block normal differentiation and cell death that confer a proliferative growth advantage. Primary strategies for leukemia therapeutics were focused on DNA damages of cancer cells with radiation or chemical drugs. Although remission can be achieved in most patients, relapse is common and long-term survival is poor for most cases, as well as the unavoidable toxicities to local norm tissues. Based on this awkward situation, an alternative strategy of inducing differentiation which consists in reprograming malignant cells to undergo terminal differentiation instead of killing them through cytotoxicity has so far gained a great interest (Tsiftsoglou et al., 2009; Sexauer et al., 2012). Till now, the best characterized clinical application of differentiation therapy is the all-trans retinoic acid-based therapy of acute promyelocytic leukemia (APL), which induces granulocytic differentiation of APL leukemic blasts, has revolutionized the therapy of this disease by converting it from a fatal to a curable one (Marchwicka et al., 2014; Takahashi et al., 2014). However, unlike the situation with APL, the development of differentiation therapy for chronic myelogenous leukemia is still far from satisfactory. Accordingly, pharmacological agents are worth exploring based on the critical mechanism of inducing differentiation. Accumulating studies have provided convincing evidences that human leukemia can be induced differentiation with some natural compounds, traditional Chinese drugs or main ingredients (Wu and Xie, 2008; Hsu et al., 2009; Zhao et al., 2012;).

Chinese angelica (Dong quai) belongs to the same plant family as parsely, celery, carrots, and poison hemlock. It traditionally has been used to treat hematologic and gynecological condition for centuries. More recently, it has been demonstrated that Angelica sinensis polysaccharide (ASP), an acetone extract polysacchaose found as the major active component in Chinese angelica, has antitumor activity across a spectrum of solid-tumors (Shang et al., 2003; Cao et al., 2010; Jin 
Lu Wang et al

et al., 2012). ASP effectively inhibit malignant brain tumors proliferation in a dose dependent manner and arrests cell cycles at the G0/G1 phase by moderating cell cycle-mediated genes expression (Tsai et al., 2005; Lee et al., 2006). ASP also has other antitumor bioactivities including senescence, pro-apoptosis, anti-angiogenesis and anti-metastasis (Cao et al., 2010; Gao et al., 2012; Liu et al., 2013). In addition, due to its fumction of immunomodulation, ASP has clinical anti-tumor efficacy to be used as a potential supplementary therapy to enhance efficacy and reduce side-effects of active cancer treatments such as chemotherapy and radiotherapy (Hui et al., 2006; Armstrong and Gilbert, 2008; Chen et al., 2010; Zhuang et al., 2012; Bunel et al., 2014).

Previous studies show that ASP has potential ability to trigger hematopoietic progenitor cells differentiation physiologically, then to facilitate normal hematopoiesis (Bradley et al., 1999; Wang et al., 2008; Lee et al., 2012). We take into account whether or not ASP can induce differentiation of leukemia cells. K562 erythroleukemia cell line, derived from a patient with chronic myelogenous leukemia in blastic crisis, has been widely used as a useful system in vitro for studying human erythroid differentiation because it expresses markers of erythroid lineage, such as hemoglobin. Here, in this report we found that ASP inhibited the proliferation of K562 cells, and induced K562 cells to undergo erythroid differentiation.

Janus kinas 2 (JAK2)/signal transducers and activators of transcription 5 (STAT5) signal transduction pathway plays critical roles in normal and leukemic hematopoiesis. JAK2/STAT5 can be activated by a plethora of cytokines that affect normal hematopoietic differentiation program, such as FL, SCF, TPO, IL-3, G-CSF, GM-CSF and EPO. EPO-induced JAK2-STAT5 phosphorylation is essential for normal erythropoiesis. Erythroid lineage development was arrested in JAK2-deficient embryos. STAT5-/embryos were found severely anemic because efficient progression of erythroid precursors through differentiation were blocked at the early erythroblast stage (Socolovsky et al., 1999; 2001; Peller et al., 2009), and stress-induced erythropoiesis was also severely impaired. Interestingly, a strong reduction of cytokine responses via JAK2/STAT5 has been shown in leukemia cells. It has been documented that negative feedback regulators modulate the JAKSTAT pathway and tightly regulate cytokine-induced signal transduction pathways by inactivation of JAKS. Thus STATS access to receptor binding sites is blocked, leading to the proteins being targeted for ubiquitination and degradation. The existence of a higher negative feedback loop may be another explanation for the block in hematopoietic differentiation of leukemia cells (Han et al., 2009; Bradley et al., 2002). In this report, we focus on the effect of ASP on both JAK2/STAT5 activation and EPO-mediated JAK2/STAT5 activation in K562 cells to determine the underlying mechanism by which ASP preferentially induces erythroid differentiation of K562 cells.

\section{Materials and Methods}

Human leukemia K562 cell line was given as a gift from the college of laboratory medicine of Chongqing medical university. Roswell Park Memorial Institute (RPMI-1640) medium and fetal bovine serum were purchased from Gibco (Gaithersburg, USA); 3-(4, 5-dimethylthiazol-2-yl)-2, 5-diphenyltetrazolium bromide (MTT) was purchased from Sigma-Aldrich (St. Louis, USA). Mouse monoclonal antibody HIR2 to Glycophorin A/B was purchased from abcam (Cambridge, USA). Rabbit Anti-JAK2, Anti-STAT5, Anti-Phospho-JAK2, Anti-Phospho-STAT5 antibodies were purchased from Cell Signaling Technology (Boston, USA). Recombinant human erythropoitin (rHuEPO) was purchased from $\mathrm{R}$ and D Systems (Minneapolis, USA). Western blotting kits were from Beyotime (Shanghai, China).

Angelica sinensis polysaccharide (ASP) was purchased from Ci Yuan Biotechnology Co., Ltd. Shanxi (Xian, China). The component monosaccharides of Angelica polysaccharide analyzed by high performance capillary electrophoresis (HPCE) were predominant arabinose, glucose, rhamnose, galactose, galacturonic acid as well as trace amount of mannose and glucuronic acid (Yang et al., 2005; 2007).

\section{Cell culture and drug treatment}

The K562 cells were cultured in RPMI-1640 supplemented with $10 \%$ fetal bovine serum and passaged every 2-3 days. Cells in their logarithmic growth phase were plated in 96 -well plates with $1 \times 10^{5}$ cells per well and divided into different groups with varying ASP concentration $0,100,200,300,500 \mathrm{mg} \bullet \mathrm{L}^{-1}$. At the different time of $1 \mathrm{~d}, 2 \mathrm{~d}, 3 \mathrm{~d}, 4 \mathrm{~d}, 5 \mathrm{~d}, 6 \mathrm{~d}$ with cells exposed to drugs, all the groups were detected by MTT Colorimetric Assay. The optimal drug concentration and duration of proliferating inhibition was acquired from the analysis of MTT Colorimetry Assay and was taken as the optimal strategy for inhibition of cell proliferation. Thereafter, K562 cells were subjected to the optimal strategy for following assays.

\section{Cell cycle analysis}

To observe cell-cycle distribution, K562 cells were treated with optional drug concentration $200 \mathrm{mg} \cdot \mathrm{L}^{-1}$ for $72 \mathrm{~h}$ in ASP treatment group and treated with RPMI-1640 supplemented with $10 \%$ fetal bovine serum in control group. All the group cells were harvested after release at the indicated times, then fixed with $70 \%$ cold ethanol and stained with propidium iodide (PI) for $30 \mathrm{~min}$ at room temperature. The fluorescent intensity of PI was analyzed with a FACS vantage SE flow cytometry (Becton Dickinson, USA) equipped with Cell Quest software (Becton Dickinson, USA).

\section{Cytochemical staining}

K562 cells were treated with ASP $200 \mathrm{mg} \mathrm{L}^{-1}$ for $72 \mathrm{~h}$ or treated with RPMI-1640 supplemented with $10 \%$ fetal bovine serum in control group for $72 \mathrm{~h}$. The cells were respectively harvested and cytospun onto slides and stained with Wright-Giemsa. The two groups cells were also subjected to benzidine staining and then visualized with a light microscope at the time point of $24 \mathrm{~h}, 72 \mathrm{~h}$. Three 
Angelica sinensis Polysaccharide Induces Erythroid Differentiation of Human Chronic Myelogenous Leukemia K562 Cells visual fields (each containing 100 cells) were randomly selected, and the percentage of benzidine positive stained cells was calculated using the image analysis system.

\section{Immunocytochemical staining}

K562 cells were treated with ASP $200 \mathrm{mg} \mathrm{L}^{-1}$ or treated with RPMI-1640 supplemented with $10 \%$ fetal bovine serum in control group. The cells were subsequently cytospun onto slides at the time point of $24 \mathrm{~h}, 72 \mathrm{~h}$. Immunocytochemical staining was performed by the primary anti-glycophorin A/B antibody (HIR2) with a 1:300 dilution and then visualized with a light microscope. Three visual fields (each containing 100 cells) were randomly selected, and the percentage of GPA positive stained cells was calculated using the image analysis system.

\section{Detection of hemoglobin by colorimetric assay}

To perform hemoglobin colorimetric assay, K562 cells were treated with RPMI-1640 supplemented with $10 \%$ fetal bovine serum in control group or optional drug concentration in ASP treatment groups for different time $24 \mathrm{~h}, 48 \mathrm{~h}, 72 \mathrm{~h}$. All the group cells were harvested and lyzed, and the concentration of hemoglobin was then determined by using a spectrophotometer.

\section{Laser scanning confocal microscope examination}

K562 cells were harvested $24 \mathrm{~h}$ after incubation with ASP $\left(0 \mathrm{mg} \bullet \mathrm{L}^{-1}, 200 \mathrm{mg} \bullet \mathrm{L}^{-1}, 500 \mathrm{mg} \bullet \mathrm{L}^{-1}\right)$. The cells were cytospun onto slides, air-dried, then fixed with ice-cold methanol for $15 \mathrm{~min}$. Blocked with $5 \%$ goat serum, the cells were incubated with primary rabbit anti-JAK2 and anti-STAT5 antibodies, and then incubated with secondary goat anti-rabbit FITC-IgG after extensive washing. The DNA was labeled with PI. The cells were viewed and photographed using a laser scanning confocal microscope (Leica TCS-NT, Germany).

Preparation of Cell lysates and Western Blotting Analysis

To perform the Western blotting assay, K562 cells were collected $24 \mathrm{~h}$ respectively after incubation with $\mathrm{ASP}(0$, $\left.100,200,300,400,500 \mathrm{mg} \cdot \mathrm{L}^{-1}\right)$. All the K562 group cells were lyzed according to Nuclear/Cytosol Fraction Protocol or Total Protein Protocol. Cytoplasmic and nuclear protein or cellular total proteins were obtained from cell lysis buffer respectively. The protein concentrations were quantified using the Bio-Rad Dc protein assay kit. 50 $\mu \mathrm{g}$ protein samples were resolved on $10 \%$ polyacrylamide gels and transferred onto $0.2 \mu \mathrm{m}$ polyvinylidence fluoride membrane. Blots were blocked with 5\% non-fat milk for $1-2 \mathrm{~h}$ at room temperature and then probed with specific antibodies Anti-JAK2, Anti-STAT5 with the ratio of 1:1000 dilution, and incubated at $4^{\circ} \mathrm{C}$ overnight. After extensive washing with TBS-T, blots were incubated with the appropriate HRP-conjugated secondary antibody for 1 $\mathrm{h}$ at room temperature. Protein bands were detected using enhanced chemiluminescent reagents.

For the phosphorylated-JAK2 and phosphorylatedSTAT5 protein detection, K562 cells were respectively treated with EPO $\left(5 \times 10^{3} \mathrm{U} \cdot \mathrm{L}^{-1}\right)$ for $2,5,30 \mathrm{~min}$ after preincubation $24 \mathrm{~h}$ in $200 \mathrm{mg} \cdot \mathrm{L}^{-1}$ ASP treatment group and

pre-incubation $24 \mathrm{~h}$ in control group. The total proteins were extracted in collected cells and then reacted with Anti-phosphorylated-JAK2, Anti-phosphorylated-STAT5 antibodies with the ratio of 1:500 dilutions. Anti-JAK2, Anti-STAT5 proteins expressions were also analyzed at the same time points by Western Blotting.

\section{Statistic analysis of the results}

Results were presented as mean \pm standard error of the mean for a given number of observations. Student's $\mathrm{t}$ test, One-way analysis of variance (ANOVA) and the Dunnett test were used to assess statistical significance using the statistical software package SPSS16.0. Results were considered statistically significant at the probability value of $p<0.05$ level.

\section{Results}

Angelica Sinensis Polysacchararide (ASP) inhibited the proliferation of $K 562$ cell line

Initial studies were performed to identify the effects of ASP on human myeloid leukemia K562 cell line using MTT colorimetric assay. As shown in Figure 1A, the proliferation of K562 cells was significantly inhibited by ASP within limits $\left(0 \sim 500 \mathrm{mg} \cdot \mathrm{L}^{-1}\right)$ in a dose and timedependent manner. Our previous studies demonstrated that ASP concentration $200 \mathrm{mg} \cdot \mathrm{L}^{-1}$ possesses the top potency of triggering normal hematopoietic stem/progenitor cells proliferation and differentiation. Thereafter, K562 cells were subjected to the safe optimal ASP concentration $200 \mathrm{mg} \cdot \mathrm{L}^{-1}$ prior to harvest to analyze cell cycles. The findings showed that the distributions of cell cycles in ASP treatment group cells were remarkably changed compared to that of the control group cells (Figure 1B, C). The cells significantly arrested at G0/G1 phase with interaction of ASP at the point of 3 day $(62.94 \pm 8.30)$ compared to control group cells $(48.91 \pm 6.45)$.

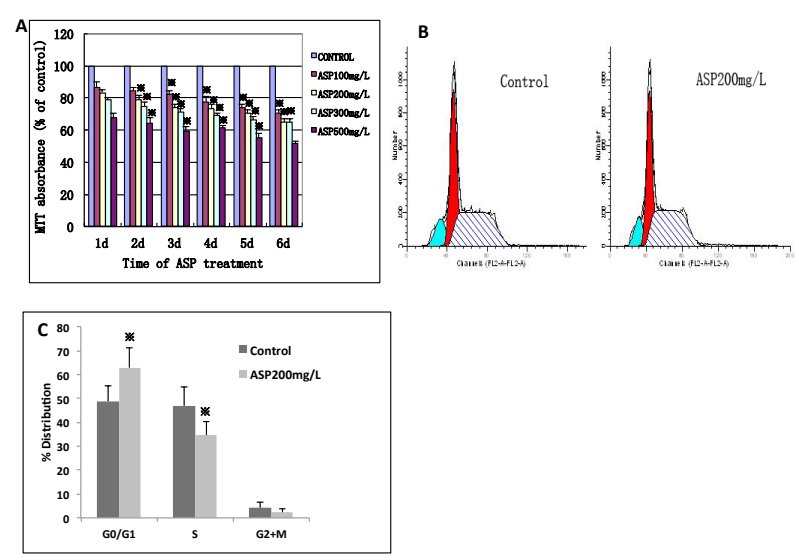

Figure 1.ASP Inhibits the Proliferation of K562 Cells. (A) MTT colorimetry assay showed that ASP dramatically inhibited K562 cells proliferation in a dose and time-dependent manner. The absorbance results of ASP-treated cells were normalized to those of non ASP-treated cells in control group. (B) ASP held K562 cells in arrest at G0/G1 phase. Representative flow cytometry graphs of cell cycle analysis of ASP-treated and control K562 cells. (C) The results of cell cycle analysis of K562 cells treated with or without ASP were presented as means \pm $\operatorname{SEM}(\mathrm{n}=3) . * p<0.05 v s$. control 
Lu Wang et al

K562 cells emerged erythoid differentiation features with induction of ASP

To determine whether ASP would functionally affect cells capable of inducing differentiation, Wright-Giemsa staining light microscopy was performed and the findings showed notable morphological changes in K562 cells as shown in Figure 2A. The cytoplasm of drug treated K562 cells with ASP $200 \mathrm{mg} \bullet \mathrm{L}^{-1}$ concentration for 3 days becomes more abundant than that of control group cells, and the nuclei to cytoplasm ratio in drug treated cells was also dramatic decreased. These typical morphological changes signed a differentiation tendency for K562 cells. To further elucidate whether the differentiation went towards erythroid lineage, the benzidine cytochemical staining and hemoglobin colorimetric analysis were performed for quantitative determination of hemoglobin in K562 cells. As shown in Figure 2, after ASP treatment, benzidine positively stained K562 cells significantly added and the level of hemoglobin in K562 cells also dramatically increased. HIR2 antigen, a classical erythroid differentiation marker, is expressed on early

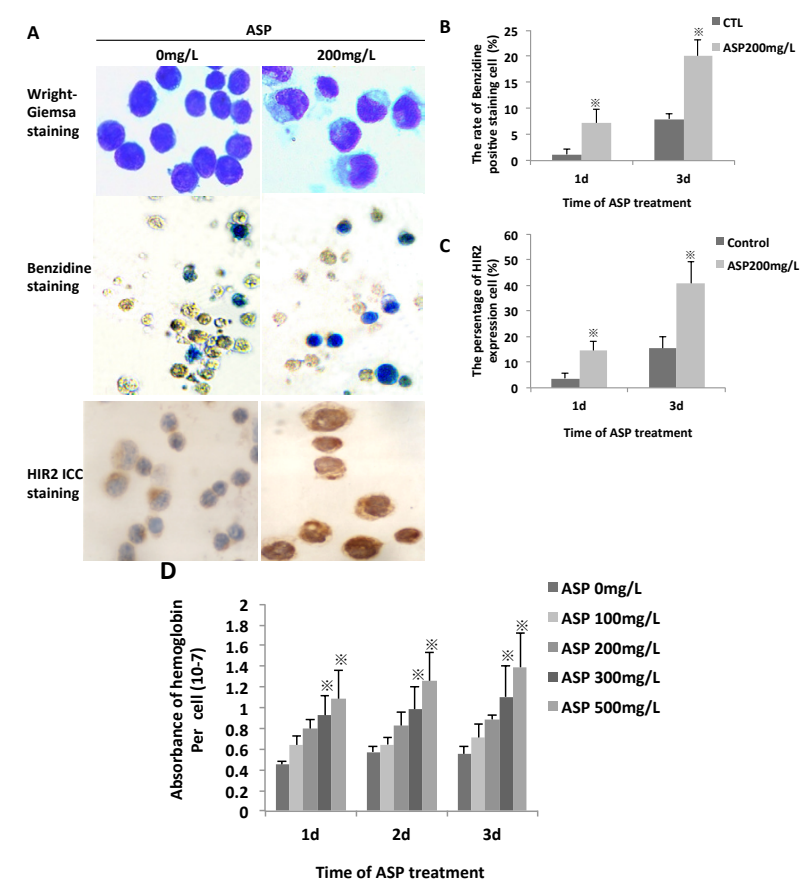

Figure 2. The Erythroid Differentiation-inducing Effect of ASP on K562 Cells. (A) After being treated with ASP $200 \mathrm{mg} / \mathrm{L}$ for 3 days, K562 cells exhibited some morphological changes evidenced by Wright -Giemsa staining with light microscopy (upper panel $\times 1000$ ) ; benzidine staining presented increased hemoglobin expression in K562 cells (middle panel $\times 400$ ); K562 cells showed enforced HIR2 expression (lower panel: left $\times 400$; right $\times 1000$ ). K562 cells were treated with RPMI-1640 supplemented with $10 \%$ fetal bovine serum in control group or with $200 \mathrm{mg} / \mathrm{L}$ ASP. (B) The percentage of benzidine positive staining cell was presented as means \pm SEM $(n=3)$ at the time point of $1 d, 3 d$. (C) The percentage of HIR2 expression cell was presented as means $\pm \operatorname{SEM}(n=3)$ at the time point of 1d,3d. (D) K562 cells were treated with RPMI-1640 supplemented with $10 \%$ fetal bovine serum in control group or with various concentration ASP $100,200,300,500 \mathrm{mg} / \mathrm{L}$ in ASP treatment groups. Colorimetric assay demonstrated the concentration of hemoglobin per K562 cell as means $\pm \operatorname{SEM}(n=3)$ at the time point of $1 \mathrm{~d}, 2 \mathrm{~d}, 3 \mathrm{~d}$. ${ }^{*} p<0.05$ vs. control erythroblasts, late erythroblasts, erythroblasts and mature erythrocytes, Belong to erythroleukemia, K562 cell line also present weak expression of HIR2. In this report we further investigate HIR2 expression in K562 cells and data showed the expression of HIR2 in $200 \mathrm{mg} \bullet \mathrm{L}^{-1}$ ASPtreated K562 cells was significantly higher than that of control evidenced by immunocytochemical staining. These findings clearly demonstrate that ASP induces K562 cells erythroid differentiation.

Activation of STAT5 is associated with the ASP erythroid inducing differentiation mechanism in $K 562$ cells

Erythropoiesis is strictly dependent on the interaction of erythropoietin (EPO) with its single transmembrane receptor erythropoietin receptor (EPOR). Binding of EPO to EPOR activates the receptor-bound tyrosine kinase JAK2 in cytoplasm and results in tyrosine phosphorylation of the EPOR cytoplasmic domain, thereby creating docking sites for downstream signaling molecules, including STAT5. STAT5 is thus tyrosine phosphorylated on recruitment to the activated receptor, then dimerizes, and migrates from cytoplasm to nucleus, where it modulates the expression of several genes involved in the control of cell proliferation and differentiation.

A
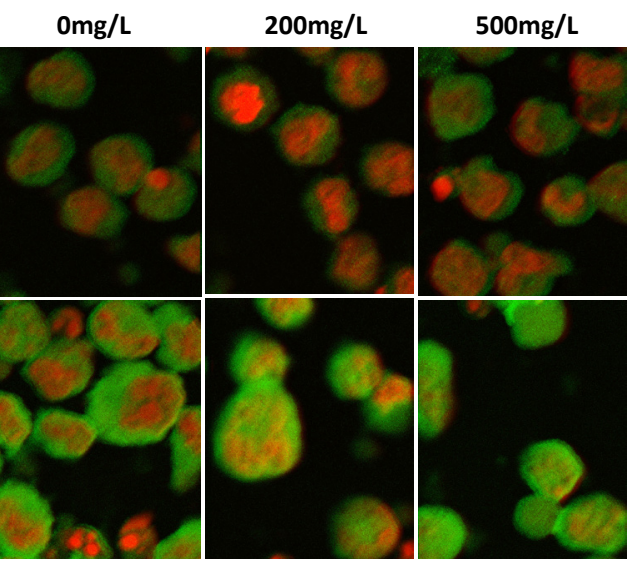

B

JAK2

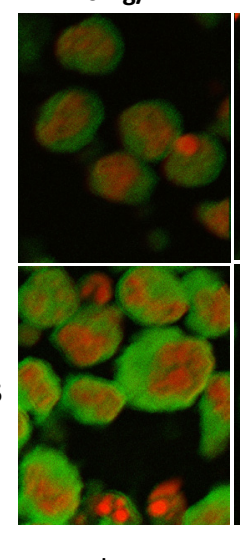

nucleus

cytoplasm

$\underset{(\mathrm{mg} / L)}{\text { ASP }}$ $\begin{array}{llllllllllll}0 & 100 & 200 & 300 & 400 & 500 & 0 & 100 & 200 & 300 & 400 & 500\end{array}$

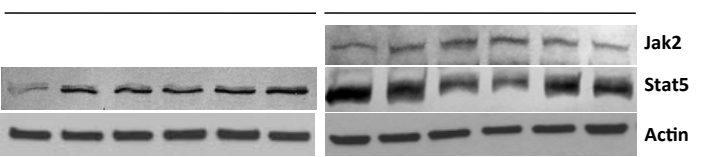

Figure 3. ASP Induces STAT5 Protein Translocation from Cytoplasm to Nuclei. K562 cells were treated with RPMI-1640 supplemented with $10 \%$ fetal bovine serum in control group or with various concentration of ASP in ASP treatment groups for $24 \mathrm{~h}$. (A) STAT5 and JAK2 protein expression in K562 cells were determined 24h by CLSM. JAK 2 protein expression in cytoplasm had no remarkable change (upper panel). ASP enforced STAT5 protein expression in nuclei (lower panel). Representative images of K562 cells treated with ASP concentration $200 \mathrm{mg} / \mathrm{L}$ and $500 \mathrm{mg} / \mathrm{L}$ are presented. Green fluorescence represents JAK2 or STAT5 protein, red fluorescence represents PI staining nucleus. (B) Western blotting assay was performed to examine JAK2 and STAT5 protein expression. JAK 2 protein expression in cytoplasm had no remarkable increase, however after ASP treatment STAT5 expression showed a dramatic increase in nuclei and decrease in cytoplasm at the same time 


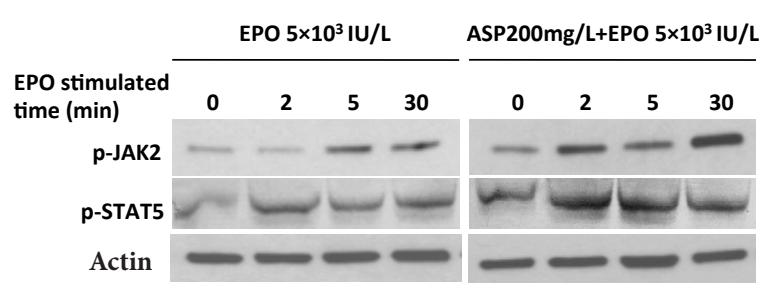

Figure 4. ASP Facilitates EPO-Mediated JAK2 and

STAT5 Activation. K562 cells were pre-incubated with or without ASP, and after $24 \mathrm{~h}$ the cells were subsequently stimulated with EPO for 2, 5, 30min, then phosphorylation of JAK2 and STAT5 in response to EPO were measured by Western blotting assay. ASP induced phospho-JAK2 and phospho-STAT5 were enhanced. Representative images of the results of protein electrophoresis of $p$-JAK2 and $p$-STAT5 are shown

Based on the erythroid inducing differentiation effects of ASP on K562 cells and the significant role of JAK2/ STAT5 on erythropoiesis, JAK2 and STAT5 proteins expression change and underlying mechanisms have been intriguing. We first analyzed the STAT5 expression in cytoplasm and nuclei of K562 cells by LSCM and western blotting assay. As observed by LSCM, the cytoplasm in the control group showed dense fluorescence intensity of STAT5, while nuclei showed weak fluorescence intensity. But after a treatment with $200 \mathrm{mg} \cdot \mathrm{L}^{-1}$ or 500 $\mathrm{mg} \cdot \mathrm{L}^{-1}$ ASP respectively for $24 \mathrm{~h}$, the distribution of STAT5 fluorescence was changed, and the fluorescent intensity became obviously enhanced in nuclei (Figure 3A). Western blotting assay also demonstrated apparent nuclear translocation of STAT5 after $24 \mathrm{~h}$ treatment of ASP. The translocation of STAT5 from cytoplasm to nuclei indicated ASP has the potential ability to activate STAT5. At the same time we detected JAK2 expression, the upstream signal molecule of STAT5. However, no significant increase of JAK2 expression in ASP-treated groups could be observed (Figure 3B).

Augment of EPO signaling is responsible for ASP-induced K562 cells erythroid differentiation

Next we examined the EPO-mediated signaling to elucidate the STAT5 activation and the erythroid lineagespecific inductive differentiation effect caused by ASP. K562 cells were pre-incubated with ASP $0 \mathrm{mg} \bullet \mathrm{L}^{-1}$ or ASP $200 \mathrm{mg} \mathrm{L}^{-1}$ for $24 \mathrm{~h}$, and then the cells were successively stimulated with EPO $5 \times 10^{3} \mathrm{IU} / \mathrm{L}$. After a treatment for $2 \mathrm{~min}, 5 \mathrm{~min}$, and $30 \mathrm{~min}$ respectively, the effect of ASP-pretreatment on EPO-induced JAK2 and STAT5 phosphorylation was detected. Western Blotting showed that after pretreatment with ASP, both of JAK2/STAT5 phosphorylation was markedly enhanced (Figure 4). These results indicated that ASP tended to facilitate JAK2/ STAT5 signal transduction by activating the response to exogenous EPO stimulation, thus promoting STAT5 translocation.

\section{Discussion}

Unlike traditional chemotherapeutic agents eliciting systemic effects on healthy tissue, induction of terminal differentiation or commitment is a conceptually attractive approach for the therapy of human leukemia. It becomes more and more highlighted that a natural product has the potential to inhibit the malignant proliferation of leukemia cells and induce them to mature differentiation, which demonstrates that Chinese herbal or active gradient may be a good candidate for differentiation inducer. Angelica sinensis, a Chinese herbal medicine, was therefore identified as a candidate for further study. Angelica sinensis polysaccharide (ASP), the main effective gradient extracted from Angelica sinensis, is known infatuatedly to be potent anti-tumor drug. Our present study demonstrates that ASP is able to inhibit the proliferation of chronic myelogenous leukemia (CML) K562 cells and the underlying mechanism is associated with erythroid inducing differentiation. It showed that growth inhibition of K562 cells was caused by $(0-500 \mathrm{mg} /$ ml) ASP in a dose and time-dependent manner. The morphological change including decreased nuclear to cytoplasm ratio after ASP treatment was observed under light microscope. Subsequently, analysis of erythroid differentiation associated markers showed that the number of benzidine and HIR2 positive staining K562 cells had been significantly increased after ASP treatment performed by cytochemical and immunocytochemical staining method respectively, and the hemoglobin concentration in ASP-treated K562 cells had also enhanced evidenced by colorimetry assay. These phenotypic changes are frequently taken as indications for cellular erythroid differentiation.

Several decades of studies have shown that EPO represents the main cytokine involved in the control of erythropoiesis (Metcalf, 2008). EPO activates JAK2/ STAT5 signaling pathway, and activation of STAT5 has been shown to be indispensable to hematopoietic progenitor cells even CD34+ stem cells proceeding normal erythropoiesis based on up-regulating BCL-XL (Kieslinger et al., 2000; Testa, 2004; Schuringa et al., 2004; Olthof et al., 2008; Grebien et al., 2008). In the present study, we first demonstrated that the JAK2/ STAT5 signaling pathway was markedly enhanced concomitant with ASP induced erythroid differentiation in K562 cells. ASP facilitated EPO- stimulating JAK2 phosphorylation, although the JAK2 total protein was not significantly changed. STAT5 protein was also markedly phosphorylated after ASP treatment, the activated STAT5 protein thus translocated from cytoplasm to nucleus. These results indicate that the JAK2/STAT5 signals play the same essential role in ASP induced erythroid differentiation in human myelocytic leukemia cells $\mathrm{K} 562$, which is in parallel with the results of other studies (Huang et al., 2009; Jerke et al., 2009; Skavland et al., 2011). The sustained tyrosine phosphorylation of JAK2 and STAT5 induces by Hexamethylenebisacetamide (HMBA) and other chemical inducers such as butyrate, can cause cell differentiation in murine erythroleukemia cell lines(Yamashita, 1998; Kawashima et al., 2001). Documents also show that human leukemia patients have proteolytic C-terminal truncated STAT5 molecules that act in a negative manner and suppress STAT5 targeted gene expression, thus blocking hematopoietic differentiation (Bunting, 2007). However, persistent activation of the STAT5 signaling facilitates differentiation. 
Malignant hematopoiesis is characterized by a lack of differentiation capacity. Although JAK2/STAT5 can be stimulated by a lot of cytokines, strongly reduced cytokine responses can be observed even in those AML characterized by constitutive STAT5 activation. It was reported that negative feedback regulators such as suppressors of cytokine signaling (SOCS) proteins were significantly higher in AML with constitutive STAT5 activation, than those without. SOCS protein negatively modulates the JAK/STAT pathway by inactivation of JAKs, blocks access of STATs to receptor binding sites and targets proteins for ubiquitination and degradation. Therefore a negative feedback loop is involved in the case of AML with constitutively activated STAT5, thus rendering those cells less sensitive to further cytokine stimulation. Based on the activation of JAK2/STAT5 signals by ASP treatment, we further addressed the problem that ASP triggers JAK2/STAT5 activation via sensitized cytokine response. Data showed that ASPpretreatment augmented the phosphorylation of JAK2/ STAT5 to ectogenic EPO stimulation. Taken together, it is conceivable that ASP has intriguing potential as an inducing differentiation agent, and ASP induced K562 cells erythroid differentiation through JAK2/STAT5 signaling pathway, which may be boosted by increased EPO response.

Interestingly, various studies have demonstrated that constitutively activated STAT5 signaling directly contributes to the oncogenesis of various myeloid leukemia due to enhanced anti-apoptotic transcription and cell-cycle progression genes (Lewis and Ward, 2008; Li et al., 2014). However, our investigation indicates that enhanced STAT5 signaling may have an opposite role in leukemia cells. This dual role phenomenon was also described in breast cancer, where STAT5 promotes both malignant transformation in mammary epithelial cells and cancer initiation. Recent new ideas have been put forward that STAT5 can inhibit tumor progression in the liver, as well as cause suppression in fibroblasts because it is able to regulate cell differentiation and senescence by activating tumor suppressors such as SOCS1, P53 and PML. Although the role of the STAT5 dual signaling mechanism is still not very clear, there are some hints from studies on normal hematopoietic progenitor cells (Ferbeyre and Morigg, 2011). Surprisingly, different phenotypes were recognized at varying levels of STAT5 activity (Wierenga, et al., 2008). High-induced activity of STAT5 resulted predominantly in differentiation towards the erythroid lineage and expansion was exhausted after three weeks of culture. Cells expressing intermediate levels of active STAT5 however, showed a more balanced differentiation program with a higher total cell output. Gene expression analysis revealed that STAT5 targets could be distinguished based upon their response to varying levels of STAT5 activity. Differentiation-related genes were found to be predominantly responsive to high levels of STAT5 activation and growth-related genes to intermediate levels of STAT5 activity.

In summary, this study demonstrates that ASP as a single agent has a potential to induce cooperating molecular events that are sufficient to induce K562 cells erythroid differentiation. Taking into account the stimulatory effects of ASP on normal hematopoiesis, there is promising for ASP to challenge the differentiation inducing therapy of chronic myelogenous leukemia.

\section{Acknowledgements}

The authors are grateful to the grant from the National Natural Science Foundation of China (No. 30572429).

\section{References}

Armstrong TS, Gilbert MR (2008). Use of complementary and alternative medical therapy by patients with primary brain tumors. Curr Neurol Neurosci Rep, 8, 264-8.

Bradley HL, Hawley TS, Bunting KD (2002). Cell intrinsic defects in cytokine responsiveness of STAT5-deficient hematopoietic stem cells. Blood, 100, 1383-89.

Bunel V, Anotoine MH, Nortier J, et al (2014). Potential nephroprotective effects of the Chinese herb Angelica sinensis against cisplatin tubulotoxicity. Pharm Biol, 11, $1-10$.

Bunting KD (2007). STAT5 signaling in normal and pathologic hematopoiesis. Front Biosci, 12, 2807-20.

Cao W, Li XQ, Wang X, et al (2010). A novel polysaccharide, isolated from Angelica sinensis (Oliv.) Diels induces the apoptosis of cervical cancer Hela cells through an intrinsic apoptotic pathway. Phytomedicine, 17, 598-605.

Cao W, Li XQ, Wang X, et al (2010). Characterizations and anti-tumor activities of three acidic polysaccharides from Angelica sinensis (Oliv.) Diels. Int J Biol Macromol, 46, 115-22.

Chen Y,Duan JA, Qian D, et al (2010). Assessment and comparison of immunoregulatory activity of four hydrosoluble fractions of Anglica sinensis in vitro on the peritoneal macrophages in ICR mice. Int Immunopharmacol, 10, 422-30.

Ferbeyre G, Morigg R (2011). The role of Stat5 transcription factors as tumor suppressors or oncogenes. Biochim Biophys Acta, 1815, 104-14,

Gao M, Zhang JH, Zhou FX, et al (2012). Angelica sinensis suppresses human lung adenocarcinoma A549 cell metastasis by regulating MMPs/TIMPs and TGF- $\beta 1$. Oncol Rep, 27, 585-93.

Grebien F, Kerenyi MA, Kovacic B, et al (2008). Stat5 activation enables erythropoiesis in the absence of EpoR and Jak2. Blood, 111, 4511-22.

Han L, Wierenga ATJ, Rozenveld-Geugien MK, et al (2009). Single-cell STAT5 signal transduction profiling in normal and leukemic stem and progenitor cell populations reveals highly distinct cytokine responses. Plos One, 4, 7989.

Hsu JW, Huang HC, Chen ST, et al (2009). Ganoderma lucidum Polysaccharides induce macrophage-like differentiation in human leukemia THP-1 cells via caspase and p53 activation. Evid Based Complement Alternat Med, 2011, 1-13.

Huang YC, Chao DK, Chao KSC, et al (2009). Oral smallmolecule tyrosine kinase inhibitor midostaurin (PKC412) inhibits growth and induces megakaryocytic differentiation in human leukemia cells. Toxicol In Vitro, 23, 979-85.

Hui MK, Wu WK, Shin VY, et al (2006). Polysaccharides from the root of Angelica sinensis protect bone marrow and gastrointestinal tissues against the cytotoxicity of cyclophosphamide in mice. Int J Med Sci, 3, 1-6.

Jerke U, Tkachuk S, Kiyanl J, et al (2009). Stat1 nuclear translocation by nucleolin upon monocyte differentiation. Plos One, 4, 8302.

Jin M, Zhao K, Huang Q, et al (2012). Isolation, structure and 
Angelica sinensis Polysaccharide Induces Erythroid Differentiation of Human Chronic Myelogenous Leukemia K562 Cells bioactivities of the polysaccharides from Angelica sinensis (Oliv.) Diels. Carbohydr Polym, 89, 713-22.

Kawashima T, Murata K, Akira S, et al (2001). STAT5 induces macrophage differentiation of M1 leukemia cells through activation of IL-6 production mediated by NF-kappaB p65. J Immunol, 167, 3652-60.

Kieslinger M, Woldman I, Moriggl R, et al (2000). Antiapoptotic activity of Stat 5 required during terminal stages of myeloid differentiation. Genes Dev, 14, 232-44,.

Lee JG, Hsieh WT, Bradley RR, et al (1999). Hematopoietic effect of Radix Angelica sinensis in a hemodialysis patient. Am J Kidney Dis, 34, 349-34.

Lee JG, Hsieh WT, Chen SU, et al (2012). Hematopoietic and myeloprotective activities of an acidic Angelica sinensis polysaccharide on human CD34+ stem cells. $J$ Ethnopharmacol, 139, 739-45.

Lee WH, Jin JS, Tsai WC, et al (2006). Biological inhibitory effects of the Chinese herb danggui on brain astrocytoma. Pathobiology, 73, 141-8.

Lewis RS and Ward ACC (2008). Stat5 as a diagnostic marker for leukemia. Expert Rev Mol Diagn, 8, 73-82.

Li J, Wei Q, Zuo GW, et al (2014). Ginsenoside Rg1 induces apoptosis through inhibition of the EpoR-mediated JAK2/ STAT5 signaling pathway in the TF-1/Epo human leukemia cell line. Asian Pac J Cancer Prev, 15, 2453-9.

Liu J, Xu CY, Cai SZ, et al (2013). Senescence effects of Angelica sinensis polysaccharide on human acute myelogenous leukemia stem and progenitor cells. Asian Pac J Cancer Prev, 14, 1-8.

Marchwicka A, Cebrat M, Sampath P, et al (2014). Perspectives of differentiation therapies of acute myeloid leukemia: the search for the molecular basis of patients' variable responses to 1,25-dihydroxyvitamin d and vitamin a analogs. Front Oncol, 4, 125.

Metcalf D (2008). Hematopoietic cytokines. Blood, 111, 485-90.

Olthof SG, Fatrai S, Drayer AL, et al (2008). Downregulation of signal transducer and activator of transcription 5 (STAT5) in CD34+ cells promotes megakaryocytic development, whereas activation of STAT5 drives erythropoiesis. Stem Cell, 26, 1732-42.

Peller S, Tabach Y, Rotschild M, et al (2009). Identification of gene networks associated with erythroid differentiation. Blood Cells Mol Dis, 43, 74-80.

Schuringa JJ, Chung KY, Morrone G, et al (2004). Constitutive activation of STAT5A promotes human hematopoietic stem cell self-renewal and erythroid differentiation. J Exp Med, 5, 623-35.

Sexauer A, Peri A, Yang X, et al (2012). Terminal myeloid differentiation in vivo is induced by FLT3 inhibition in FLT3/ ITD AML. Blood, 120, 4205-14.

Shang P, Qian AR, Yang TH, et al (2003). Experimental study of anti-tumor effects of polysaccharides from Angelica sinensis. World J. Gastroenterol, 9, 1963-1967.

Skavland J, Jorgensen KM, Hadziavdic K, et al (2011). Specific cellular signal-transduction responses to in vivo combination therapy with ATRA, valproic acid and theophylline in acute myeloid leukemia. Blood Cancer J, 1, 4 .

Socolovsky M, Fallon AEJ, Brugnara S, et al (1999). Fetal anemia and apoptosis of red cell progenitors in Stat5a-//5b-/- mice: a direct role for Stat5 in Bcl-XL induction. Cell, $98,181-91$.

Socolovsky M, Nam MDH, Fleming VH, et al (2001). Ineffective erythropoiesis in Stat5a-/-/5b-/- mice due to decreased survival of early erythroblasts. Blood, 98, 3261-73.

Takahashi H, Hatta Y, Iriyama N, et al (2014). Induced differentiation of human myeloid leukemia cells into M2 macrophages by combined treatment with retinoic acid and 